\title{
Action of Excited State Molecular Networks
}

\section{George Czerlinski ${ }^{* *}$ and Tjalling Ypma ${ }^{2}$}

${ }^{1}$ Department of Biology, Western Washington University, Bellingham, WA 98225, USA

${ }^{2}$ Department of Mathematics, Western Washington University, Bellingham, WA 98225, USA

\begin{abstract}
Nanodomains are groups of water molecules held together by an electron in an excited state. We investigate the interaction of nanodomains with living matter through acceleration of an enzyme cycle. We formulate a mechanistic model with four enzyme forms in a cycle and three successive phases. In Phase 1 a slowly catalyzing reaction approaches steady state. In Phase 2 the enzyme forms convert to their excited states using nanodomain energy, and a new stationary state is reached. The high rate of excited state energy movement in living systems leads to rapid conversion to the excited state, and the excitation energy needs to be supplied for only a short period. The excited state produces a very fast cycle, which is stable for a much smaller enzyme concentration than needed for the slow cycle. In Phase 3 the excited states decay. These phases are simulated by solving differential equations numerically.
\end{abstract}

Keywords: Enzyme reaction; Excited states; Coherence domains; Nanodomains; Sub-cellular structures

\section{Introduction}

The original concept of 'the memory of water' (see for instance Chaplin [1]) was based on water clusters serving as information carriers. However, Cowan et al. [2] showed that hydrogen bonds in these clusters have too short a lifetime to hold information. To meet this objection Czerlinski and Ypma [3] proposed the existence of nanodomains in water, formed as a result of a mechano-chemical effect such as the vigorous shaking that is part of the homeopathic process. Such nanodomains may be produced by groups of synchronously vibrating bonds in water molecules losing their vibrational energy in the process of breaking an $\mathrm{OH}$-bond. The bond breaking generates $\mathrm{H}$ - and $\mathrm{OH}$-radicals, each containing a radical electron in the triplet excited state. Czerlinski and Ypma [4] exploited the suggestion of Nagl and Popp [5] that two triplet excited states participate in an energy transfer, with one triplet becoming a singlet excited state and the other returning to the ground state. Foerster [6] and Weller [7] showed that singlet excited states produce a large $\mathrm{pK}_{\mathrm{H}}$-shift, and Smith [8] showed that $\mathrm{pH}$-changes occur when storing electromagnetic energies in water. The electron in the singlet excited state is a bound electron delocalized in the nanodomains Thus nanodomains can accept and store chemical characteristics of dissolved chemical agents, such as homeopathic substances, in the form of electromagnetic waves, when acting in coherent cooperation. The geomagnetic field promotes such cooperation. Coherent cooperation also vastly increases the lifetime of the nanodomains. Groups of nanodomains form micro domains (coherence domains) which may cooperate in organs or smaller units within a physiological network. Gather and Yun [9] recently showed that biological fluorophors, free or in live cells, can be made to act like lasers under classical conditions without damage to cells. This is relevant here, since we assume extensive coherence of waves in living systems. These domains and their electromagnetic spectra may interact with similar targets in the living system and thus be useful for medical purposes. A simplified mathematical description of the relevant kinetics together with a discussion of the associated magnetic fields was presented in Czerlinski and Ypma [10].

Czerlinski and Ypma [11] studied the interaction of aqueous nanodomains with living systems, using a model system with one enzyme catalyzed reaction. Free enzyme was assumed to be available in both active and inactive forms, with interconversion between them. The excited state energy of the aqueous nanodomains, functioning in coherent cooperation, was assumed to act on the inactive form, initiating the process of converting it to the active form via a shortlived excited state using the excitation energy derived from the fast moving nanodomains. In the present study, the excited state energy of the aqueous nanodomains is assumed to act on all the enzyme forms in a four-step catalytic cycle, converting all the enzyme components to an excited state. Although the two models differ significantly, both produce a very fast conversion, as shown here for the second model.

We study here an enzyme reaction with two catalytic cycles: a slow inner cycle involving relatively inactive enzyme forms, connected to a corresponding fast outer cycle involving their excited states. The main kinetic difference between the cycles is a pair of rate constants, which are much larger in the outer cycle than in the inner cycle. The cycles are connected by a rate constant, which is changed from zero to a large value to simulate activation of the outer cycle. Numerical simulation shows that the total enzyme concentration has to be much larger in the inner cycle than in the outer cycle in order to maintain stability in the substrate concentration values.

\section{Materials and Methods}

Since micro domains are formed by many nanodomains, many enzyme molecules are involved. These enzymes are in reactive compartments, where they occur at much higher concentration than observed in a bulk analysis (Czerlinski and Ypma [12]). The fluid volume of a cell might be such a reactive compartment. If this volume corresponds to a sphere of $10 \mu \mathrm{m}$ diameter, up to $10^{3}$ micro domains might be present. To describe the chemical kinetics involved we consider individual molecules at their respective molar concentrations and numerically simulate the system. Our focus is on the mathematical modeling of these molecular kinetics.

*Corresponding author: George Czerlinski, Department of Biology, Western Washington University, Bellingham, WA 98225,USA, Tel: 928-284-9137; Fax: 480445-9773; E-mail: gczerlinski@gmail.com

Received September 05, 2013; Accepted September 05, 2013; Published September 10, 2013

Citation: Czerlinski G, Ypma T (2013) Action of Excited State Molecular Networks. Fluid Mech Open Acc 1: 103. doi: 10.4172/2090-8369.1000103

Copyright: (c) 2013 Czerlinski G, et al. This is an open-access article distributed under the terms of the Creative Commons Attribution License, which permits unrestricted use, distribution, and reproduction in any medium, provided the original author and source are credited. 
We study an isolated enzyme reaction in the simple metabolic system of Figure 1. Component 1 represents the food source; components 2 to 5 are metabolites (substrates). Components 6 to 9 are active but electronically unexcited enzyme forms in an inner catalytic cycle, whose concentrations depend on the amount of enzyme supplied and the adjacent substrate concentrations. Components 10 to 13 are the corresponding enzyme forms changed from their ground states to singlet excited states, in an outer catalytic cycle. The rate constant $\mathrm{k}_{21}$ connects every enzyme form in the first cycle to its equivalent in the second cycle. Most of the rate constants corresponding to each other in these two cycles have the same value, except that $\mathrm{k}_{17}$ (related to $\mathrm{k}_{9}$ ) and $\mathrm{k}_{20}\left(\right.$ related to $\mathrm{k}_{12}$ ) are larger by a factor of 10 . This increase results in a much more rapid turnover in the outer enzyme cycle.

The reaction is modeled as three successive phases. Phase 1 denotes the approach to a stationary state with the initial free enzyme concentration $\mathrm{c}_{6}^{0}$ set to a nonzero value and only the inner cycle active $\left(\mathrm{k}_{21}=0\right)$. In Phase 2 the outer cycle is activated by setting $\mathrm{k}_{21}$ to a large value. The enzyme forms change from the ground states to their singlet excited states. The kinetics of this transition is described in detail below. Our simulations below show that the total enzyme concentration has to be almost ten times larger in Phase 1 than in Phase 2 in order to avoid distortions in the substrate concentration values. Excited states, even in coherence, have a limited lifetime. Phase 3 is the continuation of Phase 2 with $\mathrm{k}_{21}$ reset to zero; the small backward rate constants $\mathrm{k}_{23}$ to $\mathrm{k}_{26}$ are turned on and the excited forms revert slowly to their electronically unexcited forms. We examine two possible scenarios for this phase.

The two-step conversion from the inactive enzyme forms to their excited state is shown in the upper right corner of Figure 1 for free enzyme. There component 14 is a short-lived intermediate and component 15 is the excited state energy traveling rapidly over the system of nanodomains. We now show that the first of these two steps can safely be ignored. First we estimate a value for the diffusion limited bimolecular rate constant $\mathrm{k}_{22}$ using an equation (Czerlinski [13]) based on the work of v. Smoluchowski [14] on the aggregation of spherical

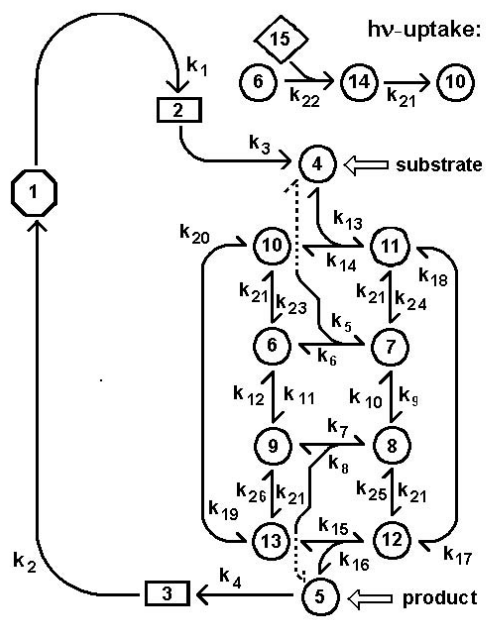

Figure 1: Reaction mechanism for a system with one enzyme reaction. Substrates/products are components 1 through 5 . Components 3 to 13 are enzyme forms with component 6 free enzyme in the ground state and component 10 free enzyme in the excited state. The excitation step involving components 6 and 10 is shown in the upper right corner as two steps, with fas bimolecular recombination first and slower structural rearrangement thereafter. Component 14 is a short-lived intermediate and component 15 is the energy component ( $h v$ in wave form or in excited state, see Discussion section). colloids. With parameters expressed in terms of moles, liters and $\mathrm{dm}$ $\left(\right.$ lliter $\left.=1 \mathrm{dm}^{3}\right)$.

$$
\mathrm{k}_{22}=4 \pi \mathrm{N}_{\mathrm{A}} \mathrm{a}_{\mathrm{D}}\left(\mathrm{D}_{1}+\mathrm{D}_{2}\right) \phi_{\mathrm{D}}
$$

where $\mathrm{N}_{\mathrm{A}}$ is Avogadro's Number ( $6 \times 10^{23}$ molecules per mole), $\mathrm{a}_{\mathrm{D}}$ is the radius of interaction (see below), $\mathrm{D}_{1}$ and $\mathrm{D}_{2}$ are the diffusion constants of the relevant components (6 and 15 in this case) and $\phi_{D}$ is an ionic factor whose value is 1 when (as here) the charge of at least one of the components is zero. Here $\mathrm{D}_{1}$, referring to enzymes, is in the range of $10^{-8}$ $\mathrm{dm}^{2} \mathrm{~s}^{-1}$, but $\mathrm{D}_{2}$ is potentially much larger. We must estimate a value for the diffusion constant of energy quanta along a chain of nanodomains. For heat diffusion in liquids a typical value is $10^{-3} \mathrm{~cm}^{2} \mathrm{~s}^{-1}$ [15]. Based on the time range for forming a nanodomain (Czerlinski and Ypma [4]) and for the onset of chemical activities (Cowan et al. [2]) we estimate the energy quanta diffusion constant to be at least 100 times faster than the heat diffusion constant, thus $10^{-3} \mathrm{dm}^{2} \mathrm{~s}^{-1}$.With these numbers we obtain $\mathrm{k}_{22}=7.536 \times 10^{13} \mathrm{M}^{-1} \mathrm{~s}^{-1}$ for $\mathrm{a}_{\mathrm{D}}=1 \mathrm{~nm}\left(10^{-8} \mathrm{dm}\right)$ and $\mathrm{k}_{22}=2.261 \mathrm{x}$ $10^{14} \mathrm{M}^{-1} \mathrm{~s}^{-1}$ for $\mathrm{a}_{\mathrm{D}}=3 \mathrm{~nm}$, covering the probable interaction range for aqueous nanodomains. The relaxation time (Czerlinski [14]) for this step (no back reaction) is given by $1 / \tau=k_{22}\left(c_{6}+c_{15}\right)$, where $c_{6}$ and $c_{15}$ represent the concentrations of components 6 and 15 respectively. With the lowest values for $\mathrm{c}_{6}$ and $\mathrm{c}_{15}$ at about $10^{-6} \mathrm{M}$ we get $1 / \tau=0.452 \times 10^{9} \mathrm{~s}^{-1}$ for the larger $a_{D}$. Since $1 / \tau$ is much larger than $k_{21}\left(10^{5} \mathrm{~s}^{-1}\right.$, Table 2 , Phase 2 ), the step with $\mathrm{k}_{21}$ controls the timing of the two-step sequence and is the only one considered further.

Similar two-step reactions start from components 7,8 and 9 to produce components 11,12 and 13 , respectively. In each case a very fast step with component 15 is followed by a much slower step controlled by $k_{21}$. As above, the fast first step may be dropped from further mechanistic consideration.

The differential equations describing the system of Figure 1 are listed in Table 1, and values for the associated constants are in Table 2. There we denote by $c$ the concentration of component $i ; c_{i}^{0}$ denotes its concentration at time $t=0$. The values in Table 2 satisfy the following conditions. The food source supplies a large excess of nutrients; thus $\mathrm{c}_{1}$ $>c_{i}$ for $i=2$ to 5 . We set $c_{1}^{0}=100 c_{i}^{0}=1 \mathrm{M}$, resulting in $c_{i}^{0}=0.01 \mathrm{M}$ for $\mathrm{i}=2$ to 5 . This implies that $\mathrm{k}_{1} / \mathrm{k}_{\mathrm{i}}=\mathrm{c}_{1}{ }^{0} / \mathrm{c}_{\mathrm{i}}^{0}=1 / 100$ for $\mathrm{i}=2$ to 5 . In the inner cycle there are two equilibration paths from component 6 to 8 . This implies the interdependence of some rate constants, namely $\mathrm{k}_{6} / \mathrm{k}_{5} \mathrm{k}_{10} /$ $\mathrm{k}_{9}=\mathrm{k}_{8} / \mathrm{k}_{7} \mathrm{k}_{11} / \mathrm{k}_{12}$. For the outer cycle similarly $\mathrm{k}_{14} / \mathrm{k}_{13} \mathrm{k}_{18} / \mathrm{k}_{17}=\mathrm{k}_{16} / \mathrm{k}_{15} \mathrm{k}_{19} / \mathrm{k}_{20}$.

The system of differential equations in Table 1 was solved

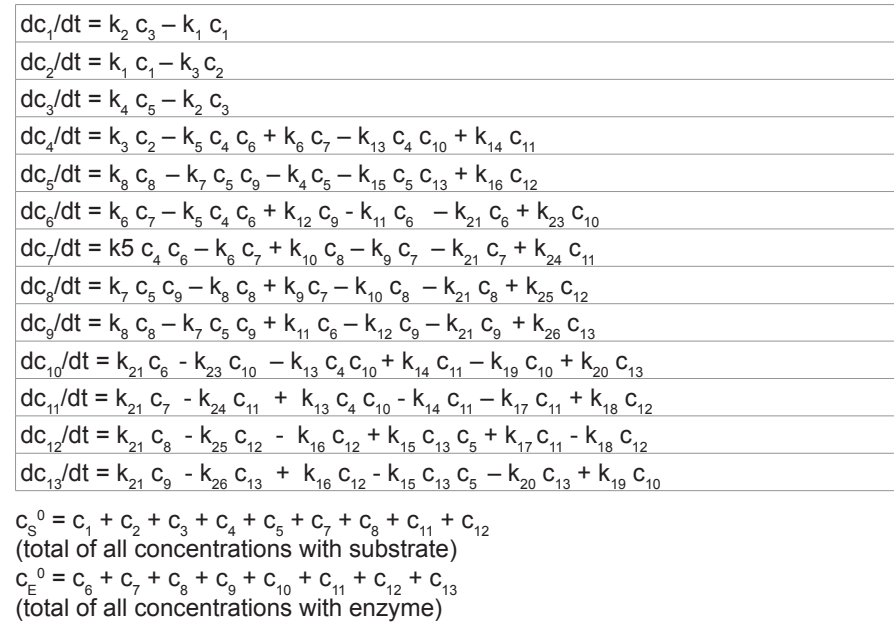

Table 1: Differential and conservation equations for the system of Figure 1. 


\begin{tabular}{|c|c|c|c|c|}
\hline Description & Symbol & Value & Units & Note \\
\hline rate constant & $\mathrm{k}_{1}$ & 0.001 & $\mathrm{~s}^{-1}$ & \\
\hline rate constants & $\mathrm{k}_{2}=\mathrm{k}_{3}=\mathrm{k}_{4}$ & 0.1 & $\mathrm{~s}^{-1}$ & \\
\hline complex 7 formation rate & $\mathrm{k}_{5}$ & $10^{6}$ & $M^{-1} s^{-1}$ & \\
\hline complex 7 dissociation rate & $\mathrm{k}_{6}$ & $10^{2}$ & $\mathrm{~s}^{-1}$ & \\
\hline complex 8 formation rate & $\mathrm{k}_{7}$ & $10^{6}$ & $M^{-1} s^{-1}$ & $=\mathrm{k}_{5}$ \\
\hline complex 8 dissociation rate & $\mathrm{k}_{8}$ & $10^{4}$ & $\mathrm{~s}^{-1}$ & \\
\hline forward isomerisation rate & $k_{9}$ & $10^{2}$ & $\mathrm{~s}^{-1}$ & \\
\hline reverse isomerisation rate & $k_{10}$ & $10^{2}$ & $\mathrm{~s}^{-1}$ & \\
\hline component 9 formation rate & $k_{11}$ & $10^{2}$ & $\mathrm{~s}^{-1}$ & $=\mathrm{k}_{10}$ \\
\hline component 6 formation rate & $\mathrm{k}_{12}$ & $10^{4}$ & $\mathrm{~s}^{-1}$ & \\
\hline complex 11 formation rate & $\mathrm{k}_{13}$ & $10^{6}$ & $M^{-1} s^{-1}$ & \\
\hline complex 11 dissociation rate & $\mathrm{k}_{14}$ & $10^{2}$ & $\mathrm{~s}^{-1}$ & \\
\hline complex 12 formation rate & $\mathrm{k}_{15}$ & $10^{6}$ & $M^{-1} s^{-1}$ & $=\mathrm{k}_{5}$ \\
\hline complex 12 dissociation rate & $\mathrm{k}_{16}$ & $10^{4}$ & $\mathrm{~s}^{-1}$ & \\
\hline forward to 12 isomer. rate & $k_{17}$ & $10^{3}$ & $\mathrm{~s}^{-1}$ & \\
\hline reverse to 11 isomer. rate & $\mathrm{k}_{18}$ & $10^{2}$ & $\mathrm{~s}^{-1}$ & \\
\hline forward to 13 isomer. rate & $k_{19}$ & $10^{2}$ & $\mathrm{~s}^{-1}$ & $=\mathrm{k}_{18}$ \\
\hline reverse to 10 isomer. rate & $k_{20}$ & $10^{5}$ & $\mathrm{~s}^{-1}$ & \\
\hline activation rate constant & $k_{21}$ & 0 & $\mathrm{~s}^{-1}$ & Phases 1, 3 \\
\hline activation rate constant & $\mathrm{k}_{21}$ & $10^{5}$ & $\mathrm{~s}^{-1}$ & Phase 2, Note 1 \\
\hline deactivation rate constants & $\mathrm{k}_{23}, \mathrm{k}_{24}, \mathrm{k}_{25}, \mathrm{k}_{26}$ & 0 & $\mathrm{~s}^{-1}$ & Phase 1,2 \\
\hline deactivation rate constants & $\mathrm{k}_{23}, \mathrm{k}_{24}, \mathrm{k}_{25}, \mathrm{k}_{26}$ & $10^{-8}$ & $\mathrm{~s}^{-1}$ & Phase 3 , case $\mathrm{A}$ \\
\hline deactivation rate constants & $\mathrm{k}_{23}$ & $10^{-8}$ & $\mathrm{~s}^{-1}$ & Phase 3 , case $B$ \\
\hline deactivation rate constant & $\mathrm{k}_{24}, \mathrm{k}_{25}, \mathrm{k}_{26}$ & $10^{-10}$ & $\mathrm{~s}^{-1}$ & Phase 3 , case $B$ \\
\hline initial substrate concentration & $\mathrm{C}_{1}{ }^{0}-25,26$ & 1 & M & \\
\hline initial substrate concentrations & $c_{i}^{0}$ & 0.01 & M & $i=2, \ldots, 5$ \\
\hline initial enzyme concentration & $\mathrm{c}_{6}{ }^{0}$ & $10^{-6}$ to $10^{-5}$ & M & see Tables 3-5 \\
\hline other initial concentrations & $c_{i}^{0}$ & 0 & M & $i=7, \ldots, 11$ \\
\hline
\end{tabular}

Note 1: Set to the value of $k 12$ of Table 2 of (Czerlinski and Ypma [3])

Table 2: Parameter values.

\begin{tabular}{|c|c|c|c|c|c|c|c|}
\hline$m$ & $c_{1}$ & $c_{2}$ & $c_{3}$ & $c_{4}$ & $c_{5}$ & $t_{\text {max }}$ & $c_{5}$ at $t_{\text {max }}$ \\
\hline 15 & 1.0092 & 0.0101 & 0.0101 & 0.0005 & 0.0101 & 20.6538 & 0.0131 \\
\hline 11.7 & 1.0079 & 0.0101 & 0.0101 & 0.0019 & 0.0101 & 34.2768 & \\
\hline 10.708 & 1.0000 & 0.0100 & 0.0100 & 0.0100 & 0.0100 & \\
\hline 9.7 & 0.9231 & 0.0092 & 0.0092 & 0.0892 & 0.0092 & \\
\hline 5 & 0.4808 & 0.0048 & 0.0048 & 0.5448 & 0.0048 & \\
\hline 2 & 0.1934 & 0.0019 & 0.0019 & 0.8408 & 0.0019 & \\
\hline 1.241 & 0.1202 & 0.0012 & 0.0012 & 0.9162 & 0.0012 & \\
\hline 1 & 0.0969 & 0.0010 & 0.0010 & 0.9402 & 0.0010 & \\
\hline$c_{i}^{0}$ & 1.0000 & 0.0100 & 0.0100 & 0.0100 & 0.0100 & \\
\hline
\end{tabular}

Table 3 : Phase 1 simulations. Stationary state concentrations at $t=10^{4} \mathrm{~s}$ for initial free enzyme concentration $\mathrm{c}_{6}{ }^{0}=\mathrm{m} \times 10^{-6} \mathrm{M}$, the time $\mathrm{t}_{\max }$ at which $\mathrm{c}_{5}$ has a maximum (if any), and that maximum value of $\mathrm{c}_{5}$.

\begin{tabular}{|c|c|c|c|c|c|c|c|}
\hline$m$ & $\mathrm{c}_{1}$ & $\mathrm{c}_{2}$ & $\mathrm{c}_{3}$ & $\mathrm{c}_{4}$ & $\mathrm{c}_{5}$ & $\mathrm{t}_{\max }$ & $\mathrm{c}_{5}$ at $\mathrm{t}_{\max }$ \\
\hline 15 & 1.0096 & 0.0101 & 0.0101 & 0.0001 & 0.0101 & 0.4508 & 0.0105 \\
\hline 11.7 & 1.0096 & 0.0101 & 0.0101 & 0.0001 & 0.0101 & 0.7112 & 0.0118 \\
\hline 10.708 & 1.0096 & 0.0101 & 0.0101 & 0.0001 & 0.0101 & 1.7258 & 0.0188 \\
\hline 9.7 & 1.0096 & 0.0101 & 0.0101 & 0.0001 & 0.0101 & 12.1339 & 0.0583 \\
\hline 5 & 1.0094 & 0.0101 & 0.0101 & 0.0003 & 0.0101 & 75.1623 & 0.0427 \\
\hline 2 & 1.0085 & 0.0101 & 0.0101 & 0.0013 & 0.0101 & 101.8591 & 0.0175 \\
\hline 1.241 & 1.0000 & 0.0100 & 0.0100 & 0.0100 & 0.0100 & 109.9006 & 0.0110 \\
\hline 1 & 0.8788 & 0.0088 & 0.0088 & 0.1348 & 0.0088 & 112.7197 & 0.0088 \\
\hline$c_{i}^{0}$ & 1.0000 & 0.0100 & 0.0100 & 0.0100 & 0.0100 & & \\
\hline
\end{tabular}

Table 4: Phase 2 simulations. Stationary state concentrations at $t=10^{4} \mathrm{~s}$ for initial free enzyme concentration $\mathrm{c}_{6}{ }^{0}=\mathrm{m} \times 10^{-6} \mathrm{M}$, the time $t_{\max }$ at which $\mathrm{c}_{5}$ has a maximum (if any), and that maximum value of $\mathrm{c}_{5}$

numerically by the Matlab (Mathworks, [16]) routine ode23s (Shampine and Reichelt [17]). This is an implementation of a variable steplength second order modified Rosenbrock method. The results of the simulations are shown in Table 3 and Figures 2 to 5 for Phase 1 and in Table 4 and Figures 6 to 9 for Phase 2; Figure 10 shows the results for Phase 3. 


\section{Results and Discussion}

\section{Kinetics}

Ideally the steady state concentrations of $c_{1}$ to $c_{5}$ match their initial values. To reach this ideal state for a particular set of rate constants in the enzyme cycle one has to select the appropriate value of the initial enzyme concentration $\mathrm{c}_{6}^{0}$. We tested several values of $\mathrm{c}_{6}^{0}$ for Phase 1 . We denote by $c_{i}^{S}$ the stationary state concentrations whose values are listed in Table 3. There is a unique value $\mathrm{c}_{6}^{0}=10.708 \times 10^{-6} \mathrm{M}$ (more precisely $10.707970574 \ldots \times 10^{-6} \mathrm{M}$ ) for which $\mathrm{c}_{\mathrm{i}}^{\mathrm{S}}=\mathrm{c}_{\mathrm{i}}^{0}$ for $\mathrm{i}=1$ to 5 and the stationary state concentration of all the substrates is the same. We call this the 'ideal' case.

Figure 2 shows the concentrations of the enzymes in Phase 1when the initial concentration of component 6 is $\mathrm{c}_{6}^{0}=15 \times 10^{-6} \mathrm{M}$. Due to the high concentration of component $4, \mathrm{c}_{6}$ decreases rapidly to form $\mathrm{c}_{7}$.

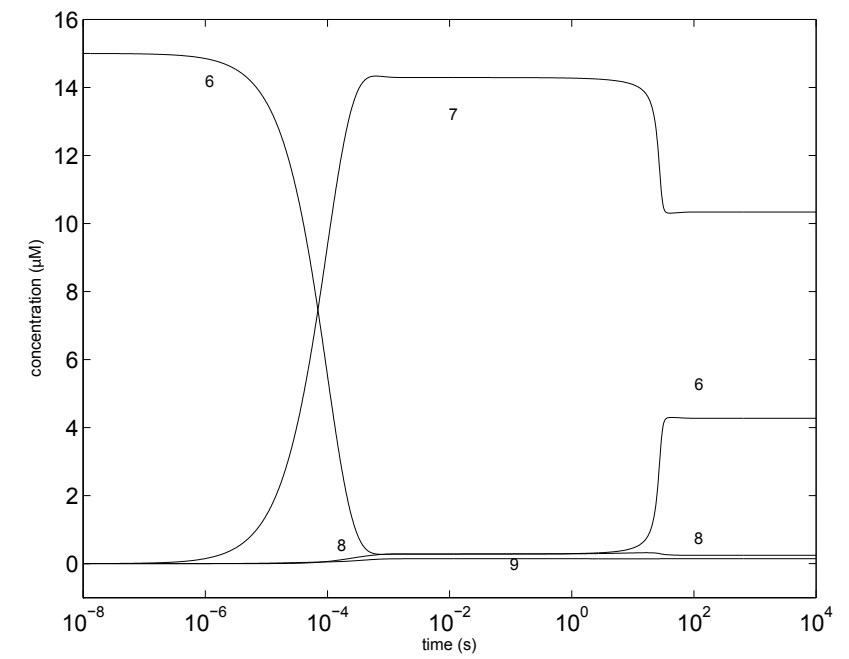

Figure 2: Phase 1 enzyme concentrations for highest initial total enzyme concentration used. Initial enzyme concentration $\mathrm{C}_{6}^{0}=15 \times 10^{-6} \mathrm{M}$, showing the concentration of enzyme components 6,7 and 8 using the differential equations of Table 1 and values of constants in Table 2.
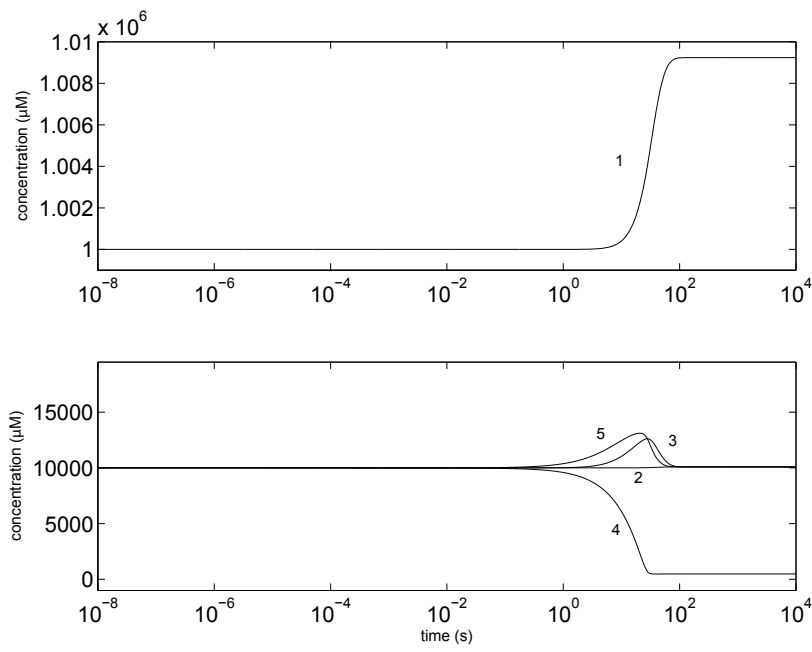

Figure 3: Phase 1 substrate concentrations for highest initial total enzyme concentration used. The concentration of components $2,3,4$ and 5 under the conditions of Figure 2.
However, the full enzyme cycle is not operational until the time range of the isomerization of component 7 to 8 is reached. Figure 3 shows the concentrations of the substrates for the same situation. The changes in components 1 and 4 essentially mirror one another, as they do in all subsequent figures. The concentrations of components 3 and 5 increase briefly.

Figure 4 shows the concentrations of the substrates for $\mathrm{c}_{6}^{0}=10.708 \times 10^{-6} \mathrm{M}$ (approximately the 'ideal' case previously defined). The changes are much smaller than in Figure 3. Here $c_{4}$ essentially reverts to its original value after its initial decrease, as do $c_{3}$ and $c_{5}$, while compensating changes in $c_{1}$ and $c_{2}$ appear only at the end.

Figure 5 shows the concentrations of most of the substrates for $\mathrm{c}_{6}^{0}=1.241 \times 10^{-6} \mathrm{M}$, which was the 'ideal' value in the sense defined above in our earlier model (Czerlinski and Ypma [3]). Interestingly, $\mathrm{c}_{4}$ changes later than $\mathrm{c}_{1}$ due to compensating effects of the other concentrations.

We see from Figures 3-5 and Table 3 that the results for Phase 1 are
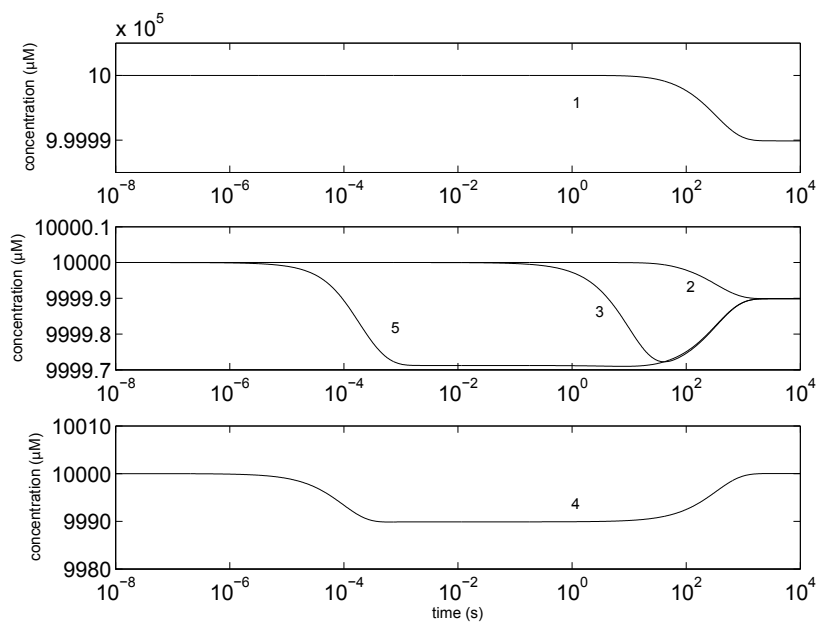

Figure 4: Phase 1 substrate concentrations for a lower initial total enzyme concentration. As Figure 3 with $\mathrm{c}_{6}^{0}=10.708 \times 10^{-6} \mathrm{M}$. Three sub graphs are needed to show all the changes.
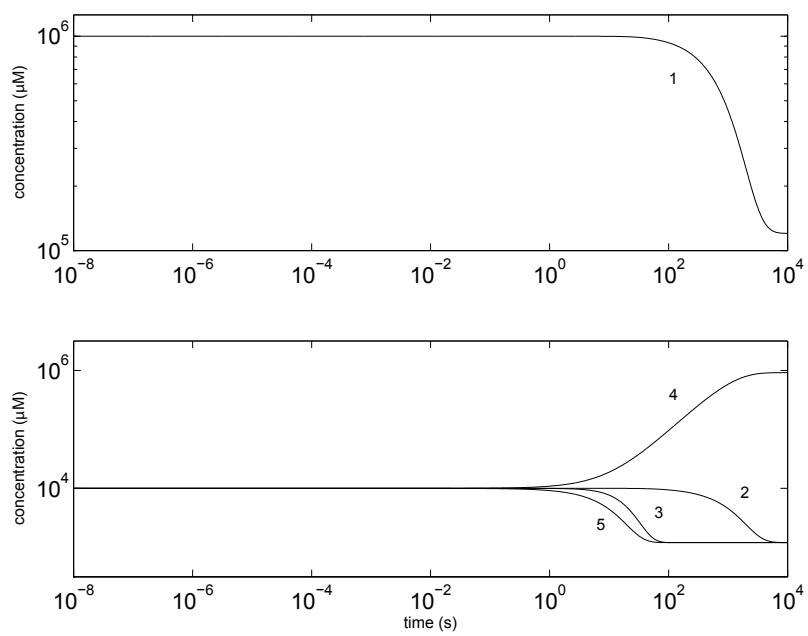

Figure 5: Phase 1 substrate concentrations for a very low initial total enzyme concentration. As Figure 3 with $\mathrm{c}_{6}^{0}=1.241 \times 10^{-6} \mathrm{M}$. Two subgraphs suffice. 
very different depending on whether $\mathrm{c}_{6}^{0}$ is above or below the 'ideal' value $c_{6}^{0}=10.708 \times 10^{-6} \mathrm{M}$. In the first case the enzyme cycle is too fast, reducing $\mathrm{c}_{4}$; in the second case the enzyme cycle is too slow, leading to an accumulation of $\mathrm{c}_{4}$. Any $\mathrm{c}_{6}^{0}>10.708 \times 10^{-6} \mathrm{M}$ eventually leads to concentrations for components 2, 3 and 5 not deviating much from that for $\mathrm{c}_{6}^{0}=10.708 \times 10^{-6} \mathrm{M}$. However, $\mathrm{c}_{6}^{0}<10.708 \times 10^{-6} \mathrm{M}$ leads to major changes in these concentrations, hampering the performance of the system.

The initial concentrations for Phase 2 are the steady state concentrations at the end of Phase 1 . Table 4 shows the values of all steady state substrate concentrations for Phase 2. Table 4 shows that for Phase 2 the 'ideal' value of $c_{6}^{0}$ (that is, the value of $c_{6}^{0}$ at the start of Phase 1 for which the substrate concentrations at the stationary state for Phase 2 are the same as at the beginning of Phase 1) is $\mathrm{c}_{6}^{0}=1.2413 \times 10^{-6}$ $\mathrm{M}$. This is the same 'ideal' value as in our prior study since identical values for the rate constants are used, but is much lower than the 'ideal' value for Phase 1 above.

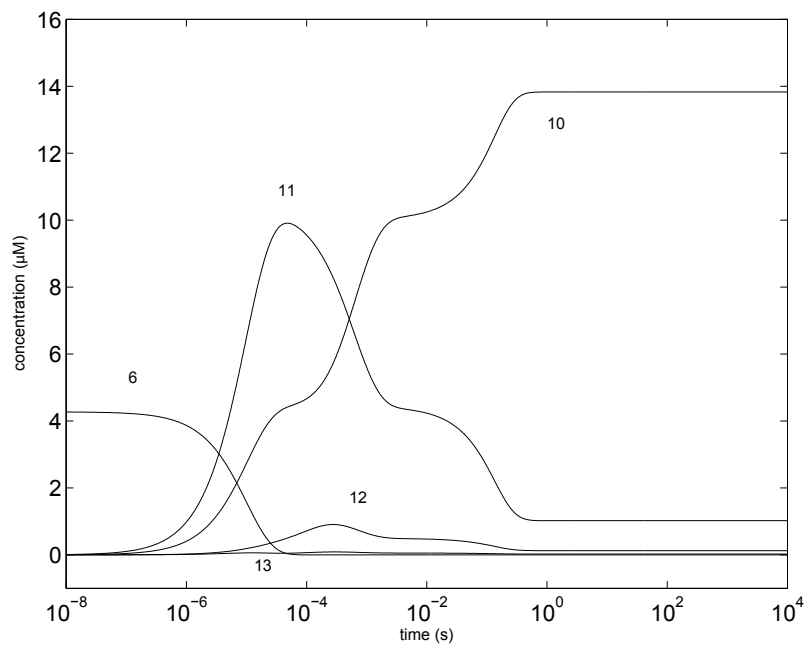

Figure 6: Phase 2 enzyme concentrations for highest initial total enzyme concentration used. Phase 2 with $\mathrm{c}_{6}^{0}=15 \times 10^{-6} \mathrm{M}$, showing the concentration of components 6 and 10 through 13 .
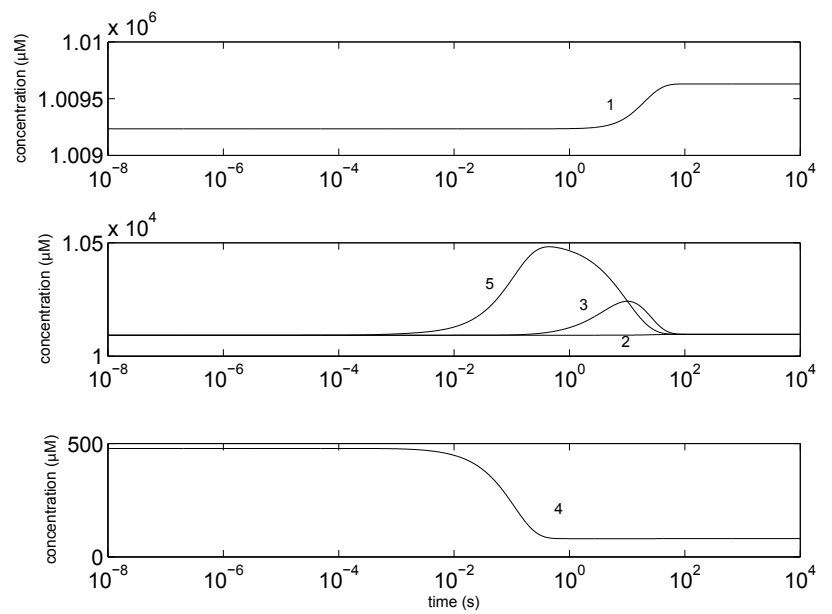

Figure 7: Phase 2 substrate concentrations for highest initial total enzyme concentration used. The concentration of components 2, 3, 4 and 5 are shown under the conditions of Figure 6 . Three subgraphs are needed to show all the changes.
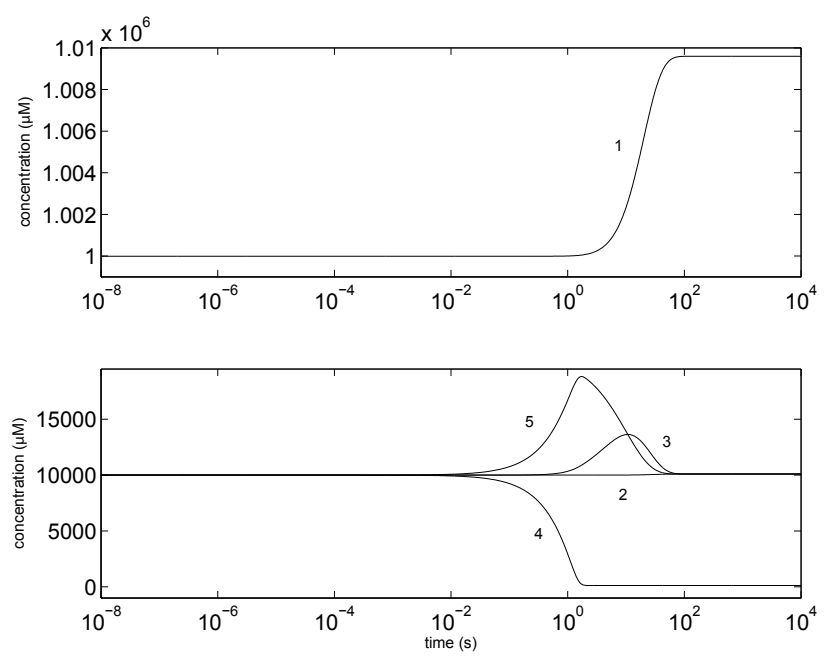

Figure 8: Phase 2 substrate concentrations for a lower initial total enzyme concentration. As Figure 7 with $\mathrm{c}_{6}^{0}=10.708 \times 10^{-6} \mathrm{M}$. Two subgraphs suffice.
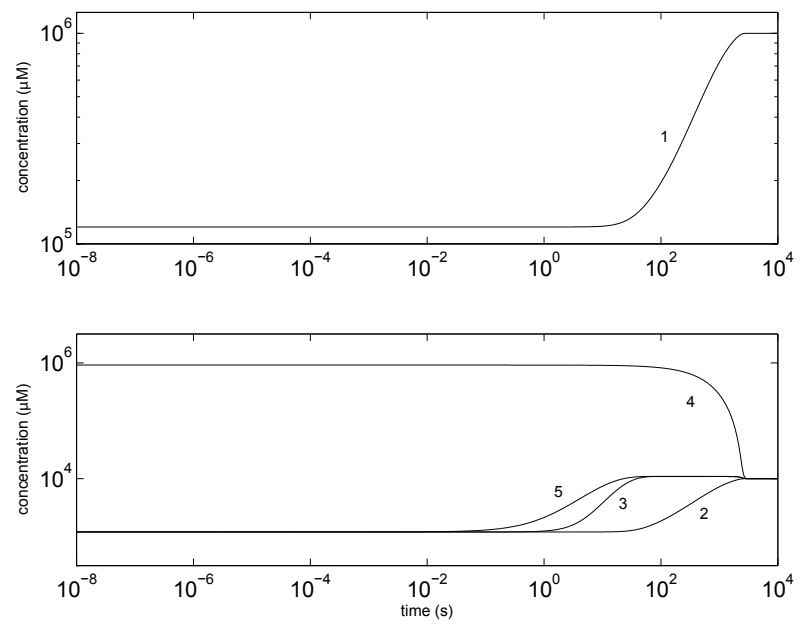

Figure 9: Phase 2 substrate concentrations for a very low initial total enzyme concentration. As Figure 8 with $\mathrm{c}_{6}^{0}=1.241 \times 10^{-6} \mathrm{M}$
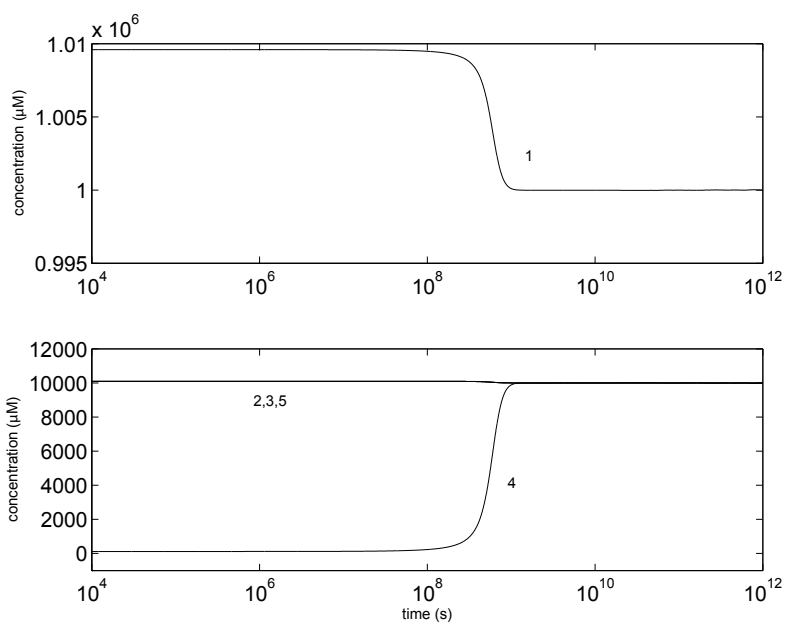

Figure 10: Phase 3 substrate concentrations; two cases. The concentration of components $1,2,3,4$ and 5 with $\mathrm{c}_{6}^{0}=10.708 \times 10^{-6} \mathrm{M}$. Only $\mathrm{c}_{1}$ and $\mathrm{c}_{4}$ differ significantly between the two cases. 

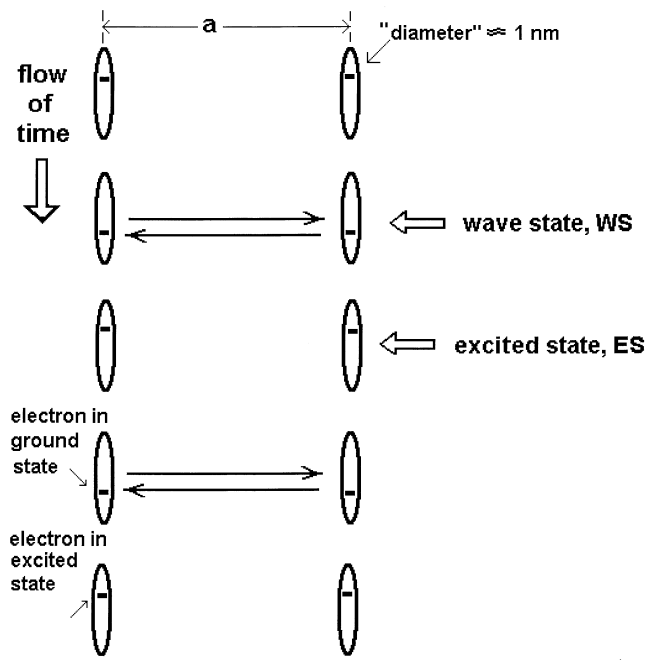

Figure 11: Description of the mechanism of coherence. A pair of cooperating excited state polymers (nanodomains) can exhibit coherent interaction upon proper orientation by the geomagnetic field. The steps of this cyclic interaction are shown from top to bottom; fs-scale. The profile of the nanodomainreflectors is shown as an ellipse, though the actual geometric structure has yet to be determined. A 'dot' near the bottom of the ellipse indicates that the delocalized electron is in its ground state $\left(\mathrm{S}^{-0}\right)$; a dot near the top indicates that the electron is in its (first) excited state $\left(\mathrm{S}^{-1}\right)$. The distances $a$ and $b$ are discussed in the text.

Figure 6 shows the concentration of enzyme components 10 through 13 for Phase 2, with $\mathrm{c}_{6}^{0}=15 \times 10^{-6} \mathrm{M}$ used for Phase 1 . Their concentrations start at zero but increase quickly as components 6 through 9 decrease. Component 11 appears early since $c_{7}$ in Figure 2 ended quite high; the conversion is determined by $\mathrm{k}_{17}$. Component 10 appears next and reacts quickly with substrate 4 to add to component 11. The stationary state levels of $c_{10}$ and $c_{11}$ in Phase 2 are quite different from those of the corresponding $\mathrm{c}_{6}$ and $\mathrm{c}_{7}$ in Phase 1.

Figure 7 shows $c_{1}$ to $c_{5}$ under the conditions of Figure 6 . The changes in $c_{1}$ and $c_{4}$ are displaced by the timing of the pulsed change in $c_{5}$.

Figure 8 shows the same components as Figure 7 but with $\mathrm{c}_{6}^{0}=10.708 \times 10^{-6} \mathrm{M}$ (the 'ideal' $\mathrm{c}_{6}^{0}$ for enzyme forms in the ground state). The steady state concentrations are distinctly different in these two figures. Both figures show a strong decrease in $\mathrm{c}_{4}$, with opposing transients in $\mathrm{c}_{3}$ and $\mathrm{c}_{5}$ displacing the timing between components 1 and 4. Figure 9 shows the same concentrations but with $\mathrm{c}_{6}^{0}=1.241 \times 10^{-6} \mathrm{M}$ (the 'ideal' $c_{6}^{0}$ for enzyme forms in the excited state). Figure 9 shows a strong decrease in $c_{4}$ from its earlier high value but the timing is visibly off due to $c_{3}$ and $c_{5}$ increasing. Evidently the conditions for Phase 2 result in a more efficient enzyme cycle, requiring much less original enzyme concentration.

Tables 3 and 4 also show the coordinates of intermediate maxima (if any) for the concentration of component 5. These maxima decrease and shift to later times with decreasing initial enzyme concentration. Table 3 shows that in Phase $1 c_{5}$ only has a transient maximum for $\mathrm{c}_{6}{ }^{0}$ above the ideal value of $\mathrm{c}_{6}^{0}=10.708 \times 10^{-6} \mathrm{M}$. Table 4 similarly shows that $c_{5}$ only has significant transient values for $\mathrm{c}_{6}^{0}$ above $1.2413 \times 10^{-6} \mathrm{M}$. Thus, the presence of transients depends on the initial value of certain concentrations at the beginning of a phase. For $\mathrm{c}_{6}^{0}=1.2413 \times 10^{-6} \mathrm{M}$ in Phase 1, $\mathrm{c}_{1}$ starts at $1 \mathrm{M}$ but has end value for Phase 1 (and hence start value for Phase 2) of $0.12 \mathrm{M}$, far below its ideal level, while $\mathrm{c}_{4}$ starts Phase 2 at $0.916 \mathrm{M}$, far above its ideal value of $0.01 \mathrm{M}$. Most of this increase in $c_{4}$ occurs at the expense of $c_{1}$.
We examined two cases for Phase 3, as reflected in alternative sets of values for the rate constants $\mathrm{k}_{23}$ to $\mathrm{k}_{26}$ listed in Table 2. In Case A all four rate constants have the same value, while in Case B one of the four rate constants is much larger than the other three. In each case a stationary state with the same stationary state values as those found in Phase 1 is reached. As shown in Figure 10, $c_{4}$ changes from a very small value to its stationary state value, but more slowly in Case B than Case A. For $c_{2}, c_{3}$ and $c_{5}$ the differences are very small.

\section{Physics}

Czerlinski and Ypma [10], Figure 4, introduced groups of nanodomains as 'polymers'; these are identical to the coherence domains of Arani et al. [18] and Del Giudice and Preparata [19]. The latter computed the radius of a coherence domain $(C D)$ as $R_{C D}=1 \mu \mathrm{m}=10^{-5} \mathrm{dm}$. Assuming a spherical volume this gives $\mathrm{V}_{\mathrm{CD}}=4.18 \times 10^{-15} \mathrm{dm}^{3}$ per $\mathrm{CD}$. Its reciprocal gives the molecular concentration as $0.239 \times 10^{15} \mathrm{CDs}$ per liter. Dividing this number by Avogadro's Number gives the concentration $\mathrm{c}_{\mathrm{CD}}=3.98 \times 10^{-10} \mathrm{M}$. Assuming maximally 1000 imprints of frequencies per CD, as obtained by Smith [20], and one frequency per nanodomain (ND), one obtains $0.239 \times 10^{18}$ CDs per liter or, with Avogadro's Number, $\mathrm{c}_{\mathrm{ND}}=3.98 \times 10^{-7} \mathrm{M}$. The assumption of tightly-packed spherical CDvolumes may be unrealistic; cubes with spherically rounded corners may be more realistic. For cubes $\mathrm{V}_{\mathrm{CD}}=8 \times 10^{-15} \mathrm{dm}^{3}$ per $\mathrm{CD}$ or $0.125 \times 10^{15}$ CDs per liter, giving $c_{C D}=2.08 \times 10^{-10} \mathrm{M}$ and $c_{\mathrm{ND}}=2.08 \times 10^{-7} \mathrm{M}$. These values of $\mathrm{c}_{\mathrm{ND}}$ are not far below our early estimate of $10^{-6} \mathrm{M}$ (Czerlinski and Ypma [4]), where we did not distinguish between bound and free radicals (bound radicals will most likely decay via free radicals).

Figure 11 shows a pair of nanodomains interacting within a $\mathrm{CD}$. The thickness depends on the number of water molecules participating in the polymerization to a nanodomain; assuming at least 4 water molecules the minimum thickness is about $1 \mathrm{~nm}$. Previously we estimated the distance 'a' between nanodomains as being at least $100 \mathrm{~nm}$, based on the requirement that for resonance to occur the distance of resonators has to be considerably above the Foerster energy transfer distance (around $10 \mathrm{~nm}$ ). Alternatively, note that coherence of electromagnetic waves extends from $200 \mathrm{~nm}$ to $800 \mathrm{~nm}$. If half the wavelength is the minimum distance for resonance between fully aligned nanodomains, then 'a' has a minimum of $100 \mathrm{~nm}$, while the longest wavelength gives a maximum value of $400 \mathrm{~nm}$.

With $\mathrm{n}=1000$ nanodomains per $\mathrm{CD}$, CDs then range in size (side length of cubes) from $1 \mu \mathrm{m}$ to $4 \mu \mathrm{m}$ (computed as $\mathrm{a} \times \mathrm{n}^{1 / 3}$ ). Since the spectrum of radio waves carried by nanodomains in a $\mathrm{CD}$ often has more than 1000 data points (Smith [20]), with one nanodomain per wavelength, CDs could be considerably larger than $4 \mu \mathrm{m}$.

Now consider the lifetimes associated with these spatial dimensions. Using the vacuum speed of light, $\mathrm{c}=3 \times 10^{8} \mathrm{~m} / \mathrm{s}$, its travel time over the above minimum distance is $\mathrm{s}=\mathrm{a} / \mathrm{c}=10^{-7} /\left(3 \times 10^{8}\right)=0.33 \times 10^{-15} \mathrm{~s}=0.33 \mathrm{fs}$ (assuming classical physics). The duration of the excited state is probably much longer than that of the wave state, at least $(\mathrm{a}(\max ) / \mathrm{a}(\mathrm{min}))^{3}=64$ times longer. Rise time and fall time for the singlet excited state are the propagation time of one wavelength each. The rapid switching between wave state and excited state extends the lifetime of the excited state, which normally is around $1 \mathrm{~ns}$ or less (Foerster [6]).

\section{Biology and coherence}

Nanodomains cooperating in CDs store electromagnetic frequencies in the UV and visible range, as in a resonator cavity, with each nanodomain in a CD storing the same frequency. For frequencies to characterize biochemical compounds a whole spectrum 
of frequencies in the radiofrequency range must be stored in one CD. Storage of different radiofrequencies in different nanodomains is assured by the Pauli principle, applied to the frequencies (as hv) stored in a cooperating $\mathrm{CD}$. CDs can thus carry the characteristic spectrum of an agent to a target in a living system [21].

Czerlinski and Ypma [11] considered living systems in which nanodomains contain DNA. Kim [22] observed linear arrays of DNA in acupuncture meridian systems, suggesting that CDs in the meridian system are linear. Then 1000 nanodomains would lead to a CD length of 0.1 to $0.4 \mathrm{~mm}$, considerably larger than ordinary cells. If the nanodomains of a cooperating pair are at each end of such a coherence domain, the travel time in the wave state could be as long as $0.4 \times 10^{-3}$ $\mathrm{m} /\left(3 \times 10^{8} \mathrm{~m} / \mathrm{s}\right)=0.133 \times 10^{-11}$ or $\mathrm{s}=1,333 \mathrm{fs}$.

Czerlinski and Ypma [4] discuss possible metal-like behavior of nanodomains, possibly corresponding to the mirrors postulated by Gariaev et al. [23] for activity in living systems. In such a metal-like reflector system there should be no absorption characteristic of radicals or similar compounds. This is exactly what Ruth and Popp [24] as well as Quickenden and Hee [25] observed.

So far only $\mathrm{OH}$-systems have been considered as forming nanodomains. The $\mathrm{H}$ component could be replaced by $\mathrm{C}$, as in ethanol or various sugars. In a biological environment, aromatic ring systems could supply the excited state and the electron in the excited state producing the metal-like environment. The principal contenders for excited state entities in proteins are tryptophan (360 nm [26]) and tyrosine (305 nm [27]). They are the only amino acids with photon absorption above the vacuum UV region (the wavelength of their emission spectrum maximum is indicated above). In a physiological system these amino-acid residues are probably aligned for coherent cooperation and form nanodomains, as aromatic amino-acid residues would do in proteins. Udenfriend and Saltzman [28] showed that fluorescence of aromatic rings in DNA only becomes apparent in hydrolysates. Their maximum fluorescence emissions are in the range of 200 to $400 \mathrm{~nm}$, where these residues could replace part of nanodomains or act as energy gates to and from nanodomains in CDs. Aromatic rings could thus act as modulators, assisting in switching spectra in and out of CDs. Such modulators may be active in low level laser treatment [29].

CDs (coherence over visible and UV-light waves) are related to the radio wave spectra mentioned earlier and the holographic waves (those around $30 \mathrm{~s}^{-1}$ ) described by Gariaev et al. [23]. Usually the frequency ranges and details of the spectra are controlled by the temperature of the environment. However, in nanodomains they are linked to the frequencies in coherence, which means that the excited states carry their energy quanta. In other words, lower frequency bands are protected from the effects of the thermodynamic heat barrier.

Statistical thermodynamics, especially a description of rotational and translational degrees of freedom, helps to understand multiple frequency bands. The quanta of translation are much smaller than those of rotation, which are those of the radiofrequencies discussed earlier. Bandwidths for the latter two ranges cannot be given, since we do not know the cellular compartment sizes involved. Spectral details could be detected on turning off the geomagnetic field and measuring released radiation. Meyl [30] describes the importance of radio waves for intercellular communication. Meyl [31] emphasizes that classical electrodynamic theory should not be applied, if events extend over less time than a single sine-wave takes. Experiments regarding the nonvalidity of classical electrodynamics could start with the correlation between the distance of pairs of nanodomains in coherence and the concentration of these nanodomains, $\mathrm{c}_{\mathrm{ND}}$.

\section{Verification}

The analysis of Endler et al. [32] on the climbing speed of frogs as a function of low-level thyroxin concentration in their water showed that (i) succession (vigorous shaking) of the hormone solution after each dilution is required for any effect, (ii) the effect is maximal even if only a few drops of a sample are added to the water, with the drops diluted in thyroxin to much less than 1 molecule per liter, supporting the action of homeopathic dilutions, (iii) if a solution sample is sealed in a glass ampoule and placed into the bowl with the frogs, the frogs are almost as active as without the ampoule encasement of the hormone, suggesting that electromagnetic radiation is involved. We lack details of the electromagnetic spectra emitted from these vials. Nevertheless these and other data led Czerkinski and Ypma [3] to their molecular description of high-dilution homeopathy, where succession produces water radicals, with some bound in small polymers carrying the electromagnetic information.

How this information is carried was elucidated by the $\mathrm{pH}$ experiments of Scott-Morley [33] and Smith [8]. The former placed homeopathic solutions in ampoules in front of a wideband radio wave amplifier, collecting the amplified radiation in (pretreated) water, and then measured the $\mathrm{pH}$-change, which was significantly above noise level. The latter simulated the storage action of homeopathic solutions by using a radio wave generator, stepping one frequency at a time. $\mathrm{He}$ found that at $\mathrm{pH} 8$ he could only saturate the water with close to 400 frequencies, producing a distinct $\mathrm{pH}$-change of 0.04 units. When the geomagnetic field was reduced to $1 / 100$ of its initial value, by placing the solution temporarily into a mu-metal box, the $\mathrm{pH}$-change reversed, indicating the loss of photons. Unfortunately neither author calibrated their $\mathrm{pH}$-change by adding a known amount of $\mathrm{HCl}$ or $\mathrm{NaOH}$. Nevertheless these experiments show that singlet excited states are involved in storing electromagnetic energy in nanodomains. Since $\mathrm{pH}$-changes are based on the absorption of electromagnetic radiation, the spectra of such radiation should contain information on the origin of the radiation. Such changes arise from the difference in the $\mathrm{pK}_{\mathrm{H}}$ of ordinary water compared to when $\mathrm{H}_{2} \mathrm{O}$ is bound in the nanodomain with the radical electron in the excited state. Since the excited state derives from photon absorption, these photons could be observed from the output of a wideband amplifier with input restricted to a specific area of the living system. Variation of the magnetic field strength may be useful.

The rate of diffusion of excited state energy quanta is difficult to measure since it involves fast optical transfer of energy. The concentration of excited state energy quanta might be measured by rapidly moving a mu-metal box over samples, thereby reducing the effect of the earth's magnetic field to near zero, and observing the emitted photons and the time course of the emission. The distance between nanodomains with excited state energies could possibly be determined by photoflash luminescence using lasers, thus verifying these distances independent of volume estimates and obtaining a better value for nanodomain concentrations.

Another approach to measuring the concentration of nanodomains is to observe free and bound radicals directly. Zafiriou et al. [34] previously used NO and NO-derivatives to measure radicals. Different NO-derivatives may form different complexes, thus revealing differentiating spectral characteristics including those of radical electrons bound within nanodomains. 


\section{Conclusions}

We described an enzyme activation process which may occur throughout a living system: classical enzyme reactions are energized by converting enzyme components to their excited state, which in turn increases values of rate constants and speeds up turnover. Since nanodomains cooperate coherently in coherence domains they are long-lived, with the electromagnetic spectrum of the coherence domains controlling the action of the stored energies. These domains may interact with similarly designed targets in living systems. These domains may thus be medically useful, as may the electromagnetic spectra themselves. Considering nanodomains as electromagnetic energy storage elements also introduces the notion of photon communication within living systems.

\section{Acknowledgement}

We thank Submicron Inc for contributing to the article processing charges. George Czerlinski is founder and president of Submicron Inc.

\section{References}

1. Chaplin M (2007) The memory of water an overview Homeopathy 96: 143-150.

2. Cowan ML, Bruner BD, Huse N, Dwyer JR, Chugh B, et al. (2005) Ultrafast memory loss and energy redistribution in the hydrogen bond network of liquid H2O Nature 434: 199-202.

3. Czerlinski G, Ypma T (2010) Domains of water molecules provide mechanisms of potentization in homeopathy Water 2: 1-14.

4. Czerlinski G, Ypma T (2011) Stabilization of aqueous electrometric nanodomains. J Comput Theor Nanoscience 8: 1400-1408.

5. Nagl W, Popp FA (1983) A physical (electromagnetic) model of differentiation Basic considerations. Cytobios 37: 45-62.

6. Foerster TH (1950) Electrolytic dissociation of excited molecules. Z Electrochem Angew Physics Chem 54: 42-46.

7. Weller A (1955) The reaction mechanism of acid dissociation as an example of the protolytic reaction of excited beta-naphthol Z physikal Chemie NF 3 : 238-254.

8. Smith CW (2007) Water - its clinical and scientific depth. Emoto M The Healing Power of Water. Carlsbad CA Hay House London 77-88.

9. Gather NC, Yun SH (2011) Single-cell biological lasers. Nature Photonics 5: 438.

10. Czerlinski G, Ypma T (2011) Homeopathic potentization based on nanoscale domains. Journal of Alternative and Complementary Medicine 17: 1165-1173.

11. Czerlinski G, Ypma T (2012) The targets of information-carrying nanodomains J Nanosci Nanotechnol 12: 2239-2247.

12. Czerlinski G, Ypma T (2008) Dimensional effects on single molecule kinetics in submicron vacuoles.J Bionanosci 2: 19-26.

13. Czerlinski G (1966) Chemical Relaxation. An Introduction to Theory and Application of Stepwise Perturbation Marcel Dekker, New York.

14. Smoluchowski M (1917) Attempt toward a mathematical description of the kinetics of coagulation of colloids in solution. Z Physikal chemistry 92: 129-168.

15. http://www.pojman.com/mg materials/Diffusion/Diffusion.html

16. The Mathworks (2008) Matlab 8.0 Natick, MA.

17. Shampine LF, Reichelt MW (1997) The Matlab ODE suite. SIAM J Sci Comput. 18: $1-22$.

18. Arani R, Bono I, Del Giudice E, Preparata G (1995) QED coherence and the thermodynamics of water. Int J Mod Phys 9: 1813-1841.

19. Del Giudice E, Preparata G, Sassaroli E, Srivastava Y, Swain J, et al. (1998) A new QED picture of water Understanding a few fascinating phenomena
"Macroscopic Quantum Coherence". World Scientific Hackensack, NJ, USA, 108-129.

20. Smith CW, Hyland GJ, Rowlands P (2008) Froehlich's interpretation of biology through theoretical physics Herbert Fröhlich FRS A physicist ahead of his time. University of Liverpool (2ndedn) 107-154.

21. Czerlinski G, Ypma T (2008) Dimensional effects on single molecule kinetics in submicron vacuoles.J Bionanosci 2: 19-26.

22. Kim BH (1964) On the kyungrak system. J. Acad. Med. Sci. DPR Korea. English version Foreign Languages Publishing House Byongyank.

23. Gariaev PP, Birshtein BI, larochenko AM, Marcer PJ, Tertishny GG, et al. (2002) The DNA-wave biocomputer.

24. Ruth E, Popp FA (1976) Experimental studies on the ultra-weak photon emission of biological systems. Naturforsch 31: 741-745.

25. Quickenden TE, Hee SQ (1976) The spectral distribution of the luminescence emitted during growth of the yeast saccharimyces cerevistae and its relationship to mitogenetic radiation. Photochem Photobiol 23: 201-204.

26. Prahl S, Tryptophan. http://omlc.ogi.edu/spectra/PhotochemCAD/html/tryptophan.html

27. Prahl S, Tyrosine. http://omlc.ogi.edu/spectra/PhotochemCAD/html/tyrosine.html

28. Udenfriend S, Zaltzman P (1962) Fluorescense characteristics of purines pyrimidines and their derivatives Measurement of guanine in nucleic acid hydrolysates. Analyt. Biochem 3: 49-59.

29. Huang YY, Hamblin M, Chen ACH (2009) Low-level laser therapy. an emerging clinical paradigm.

30. Meyl K (2011) DNA and Cell Resonance. INDEL G m b H Verlagsabteilkung Villingen-Schwenningen, (2ndedn), Germany.

31. Meyl K (2011) "Scalar wave technique" with excepts from the 3rd Banzur Electro-Magnetic Tolerance and documentation for the demonstration set fot the transmission of electric scalars, INDEL Verlag, (2ndedn) Germany.

32. Endler PC, Pongratz W, Smith CW, Schulte J (1995) Non-molecular information transfer from thyroxine to frogs with regard to homeopathic toxicology. Vet Human Toxicol 37: 259-260.

33. Scott-Morley A, Anfang G (2010) The ultimate cure of diseases.

34. Zafiriou OC, Blough NV, Micinski E, Dister B, Kieber D, et.al. (1990) Molecular probe systems for reactive transients in natural waters. Marine Chemistry 30 45-70. 